\title{
Microstructural analysis of subchondral bone in knee osteoarthritis
}

\section{L.A. Holzer ${ }^{1,7}$ - M. Kraiger ${ }^{2}$ - E. Talakic $^{3}$ - G.A. Fritz ${ }^{3}$ - A. Avian ${ }^{4}$ - A. Hofmeister ${ }^{5}$ - A. Leithner ${ }^{1}$ - G. Holzer ${ }^{6}$}

Received: 9 January 2020 / Accepted: 7 May 2020 / Published online: 29 May 2020

(C) The Author(s) 2020

\begin{abstract}
Summary The results of this study show increased formation of bone in the subchondral areas in advanced stages of osteoarthritis of the knee. These changes seem to be influenced by mechanical factors.

Introduction Subchondral bone changes seem to contribute to the progression of knee osteoarthritis (OA). This study aimed to analyze subchondral bone microstructure in specimens of late-stage knee OA in respect to articular cartilage damage, meniscus integrity, and knee joint alignment.

Methods Thirty proximal tibiae of 30 patients (20 female and 10 male) with late-stage OA retrieved during total knee arthroplasty were scanned using a high-resolution micro-computed tomography. The scans were semi-automatically segmented into five volumes of interest. The volumes of interest were then further analyzed using commercially available software. The degree of articular cartilage damage was assessed semi-quantitatively by magnetic resonance imaging before surgery.

Results The mean bone fraction volume (bone volume/total volume (BV/TV)) in all weight-bearing locations was significantly higher compared to the non-weight-bearing reference point below the anterior cruciate ligament $(p=0.000)$. The mean BV/TV in the medial compartment was significantly higher compared to the lateral compartment $(p=0.007)$. As for the BV/TV in intact menisci, there was a significantly lower subchondral bone fraction volume compared to subluxated or luxated menisci in the medial $(p=0.020)$ and lateral compartment $(p=0.005)$. Varus alignment had a significantly higher subchondral BV/TV in the medial compartment, whereas valgus alignment had a significantly higher subchondral BV/TV in the lateral compartment $(p=$ 0.011 ).

Conclusions The results show significant differences of subchondral bone microstructural parameters in respect to cartilage damage, meniscus' structural integrity, and knee joint alignment. Therefore, subchondral bone changes seem to be a secondary process in the late-stage OA of the knee caused by mechanical changes.
\end{abstract}

Keywords Bone microstructure $\cdot$ MicroCT $\cdot$ MRI $\cdot$ Osteoarthritis $\cdot$ Subchondral bone

\section{L.A. Holzer}

lukas.holzer@auva.at

M. Kraiger

m.kraiger@tugraz.at

E. Talakic

emina.talakic@medunigraz.at

G.A. Fritz

geraldalois.fritz@klinikum-graz.at

A. Avian

alexander.avian@medunigraz.at

A. Hofmeister

a.hofmeister@medunigraz.at

A. Leithner

andreas.leithner@medunigraz.at
G. Holzer

gerold.holzer@meduniwien.ac.at

1 Department of Orthopaedics and Trauma, Medical University of Graz, Auenbruggerplatz 5, 8036 Graz, Austria

2 Institute of Medical Engineering, Graz University of Technology, Graz, Austria

3 Division of General Radiology, Department of Radiology, Medical University of Graz, Graz, Austria

4 Institute for Medical Informatics, Statistics and Documentation, Medical University of Graz, Graz, Austria

5 PreClinical Imaging Group, Center for Biomedical Research, Medical University Graz, Graz, Austria

6 Department of Orthopaedics and Trauma Surgery, Medical University of Vienna, Vienna, Austria

7 AUVA Trauma Center Klagenfurt, Waidmannsdorfer Straße 35, Klagenfurt am Wörthersee, Austria 


\section{Introduction}

Osteoarthritis $(\mathrm{OA})$ is a chronic disease characterized by the deterioration of joint cartilage causing pain, stiffness, and impaired range of motion (ROM) [1].

Subchondral bone is important for physiological joint function. The close relationship between subchondral bone and joint cartilage make both a functional unit. Both tissues are mechanically and biologically connected. Mechanical or biological changes of subchondral bone will affect cartilage and vice versa [2]. The tissue of origin, however, has been discussed controversially.

The roles of subchondral bone in the articular joint are to distribute forces, prevent stress concentrations, and adapt to maintain conformation of the joint [3]. Articular cartilage is designed for loadbearing. Its high water content allows deformation under compressive loads without failure. Its capacity to withstand tension or shear stresses that occur at the edges of joint contact regions is lower. This could be exacerbated in homogeneously dense and stiff areas where the cartilage would deform more in regions overlying less dense parts of the subchondral bone than in denser regions. The regions between these stiff and less stiff regions are sites of stress concentration at which the cartilage is more likely to fail under tension conditions [3].

In preclinical experimental studies, it was shown that mechanical loading contributes to OA progression by affecting the subchondral bone [4]. It is believed that due to chronic impacts associated with microtraumas (microfractures) in the subchondral bone, increased remodeling is initiated.

In late-stage OA, the chronic (microfracture-) healing process results in an imbalance of remodeling in favor of bone formation leading to sclerosis and thickening of the subchondral bone. Subchondral sclerosis is a classic sign of the progression of OA which can be seen in X-rays [5]. Its role in the progression of OA has been discussed controversially, whether it initiates OA progression or can be seen as a consequence of the catabolism of the articular cartilage $[3,5,6]$.

Data is accumulating that subchondral bone changes may be involved in the progression of OA [2, 3, 6]. Experimental as well as clinical studies showed a correlation of cartilage damage and subchondral bone density [3, 6-8]. Microstructural analyses have been performed in human specimens of OA of the hip as well as OA of the knee. In both anatomic regions, increased subchondral bone density beneath areas of cartilage damage has been found [8].

In an experimental biomechanical study, it was demonstrated that cartilage in OA has less potential of shock absorption compared to normal physiological knee cartilage [9]. Furthermore, the loss of a meniscus' integrity by either "meniscus injury" or "total meniscectomy" resulted in an increased impact force of $113 \%$ up to $121 \%$ in "total meniscectomy." Therefore, the resulting increased impact in the subchondral bone will result in sclerotic bone after microfracture healing [9].

Knee alignments as well as meniscus' integrity are structural and geometric parameters that influence mechanical loading in the knee joint. Varus alignment for example leads to higher loading conditions of the medial compartment. An intact meniscal structure prevents the cartilage to degenerate $[10,11]$. This is of major interest in clinical practice where resection of meniscus in case of tears and the effect in patients with OA is discussed controversially $[12,13]$.

We aimed to analyze subchondral bone microarchitecture in respect to cartilage damage, meniscus' integrity and knee joint alignment in proximal tibia specimens of patients with late-stage OA. We hypothesized that (1) subchondral bone beneath areas with a high degree of cartilage damage may have increased bone density compared to areas with lower degrees of cartilage damage and (2) intact meniscal structures may have reduced subchondral bone density compared to luxated or subluxated ones and that knee joint malalignment may cause higher density in the subchondral bone of the affected compartment. To our knowledge, this is the first study on subchondral bone changes conducted in patients with advanced stage of OA of the knee that uses a combination of approach and methods to compare subchondral bone microarchitecture with cartilage damage, meniscus' integrity, and knee joint alignment.

\section{Methods}

\section{Patients}

Thirty consecutive patients ( 20 female and 10 male, mean age $70.4 \pm 9.9$ years) who were scheduled for total knee arthroplasty (TKA) were prospectively chronologically (sequentially) included in this study. Inclusion criteria were age between 50 and 90 years, either female or male sex, and the presence of primary late-stage OA of the knee (Kellgren and Lawrence classification grade 3 or 4). Exclusion criteria were the presence of benign or malignant tumor in the affected joint, local or systemic metabolic bone disorders (except osteoporosis), rheumatoid arthritis, prior trauma, or surgery of the joint (except arthroscopy).

\section{Clinical assessment}

Patients were recruited and evaluated at our department's outpatient clinic and subsequently scheduled for TKA. One day prior to surgery, the patients were examined physically and assessed using a standardized questionnaire, the Knee Society Score (KSS) at the ward [14].

The KSS was developed by consensus of the Knee Society [14]. The KSS is a widely used functional 
outcome score for knee arthroplasty. The KSS consists of two parts, the Knee Score and the Function Score. A maximum of 100 points can be obtained in both scores each.

\section{Demographic data and laboratory blood analyses}

Demographic and medical data was retrieved from our records. Patients had routine laboratory blood analyses 1 day prior surgery.

\section{X-rays}

Preoperatively, all patients had X-rays of the knee joint in anterior/posterior and lateral view, axial patella "skyline view" (Merchant's view), and a full-leg X-ray to analyze the degree of malalignment.

\section{Magnetic resonance imaging (MRI)}

Preoperatively, within 6 weeks before TKA, patients had a MRI scan of the affected knee joint. MR images were obtained using a 3.0 Tesla MRI scanner with a maximum gradient amplitude of $40 \mathrm{mT} / \mathrm{m}$ (Siemens Magnetom Trio ${ }^{\circledR}$, Siemens AG, Erlangen, Germany). The following protocols were used: a transversal T2-weighted 3D CISS (Constructive Interference in Steady State), a sagittal 3D GRE (Gradient recalled Echo), and a sagittal 3D DESS (Dual Echo Steady State).

The severity of OA was estimated by using MRI scans by two board-certified radiologists (E.T., H.S.) using a semiquantitative scoring system published by Yuslih et al. [15]. The scores are between 1 and 4, with 4 indicating an advanced stage of OA. Moreover, the integrity of both the medial and the lateral menisci was also assessed by MRI scanning. Menisci were defined either as "intact meniscus" (degenerative, but normal structure) or "non-intact meniscus" (luxated or subluxated).

\section{Specimens}

Proximal tibial specimens were retrieved during TKA. In TKA, the tibial plateau is resected by a maximum of $10 \mathrm{~mm}$ below the joint line in one piece with the help of a standardized cutting guide in order to preserve as much bone stock as possible. The specimens were fixed in ethanol $70 \%$ (Pharmacy Landeskrankenhaus - Universitätsklinikum Graz, Graz, Austria) and stored at $-20^{\circ} \mathrm{C}$ until analysis. Before $\mu \mathrm{CT}$ analysis, specimens were thawed for $24 \mathrm{~h}$.

\section{Micro-computed tomography $(\mu \mathrm{CT})$ imaging and image processing}

\section{$\mu \mathrm{CT}$ imaging}

The specimens were scanned using a preclinical MicroCT system (Inveon MicroCT ${ }^{\circledR}$, Siemens AG, Erlangen, Germany). A scan of $360^{\circ}$ was made at a voltage of $80 \mathrm{kVp}$ and a current of $500 \mu \mathrm{A}$ and an exposure time of $1300 \mathrm{~ms}$. The effective pixel size was $43.96 \mu \mathrm{m}$. In addition, the specimens were consistently oriented in the scanner and aligned with the horizontal axis of the scanner. The specimens were positioned on a soft cotton tissue to reduce movement during the scan.

\section{Image segmentation and trabecular bone processing}

The segmentation of the three-dimensional (3D) cubic VOIs was performed using an in-house imaging software developed in MATLAB Version 8.2 R2013b (The MathWorks Inc., Natick, MA, USA). In each tibial bone sample, five different cubic volumes with an isotropic dimension of $4 \mathrm{~mm}^{3}$ were defined on the median coronal axis: lateral (submeniscal) (1), lateral compartment - center (2), center of the tibia as a reference (in between the tibial footprint of the ACL and PCL) (3), medial compartment-center (4), and medial (submeniscal) (5) VOI [see Fig. 1a]. These compartments were segmented according to the following processing steps.

At first, a preliminary threshold was used to extract the mineralized bone phase. Each coronal and sagittal slice of the resulting binary data was superimposed onto a single coronal and sagittal plane depicting the outermost contour of the sample. Within the resulting coronal plane, a rectangular region was selected covering the majority of the cancellous bone starting with the positioning of the VOIs within the coronal plane [Fig. 1a]. The sides of the oblong were aligned parallel to the image margins. The width of the rectangle in mediallateral direction was defined at the level of the median. At this level, a gap of $4 \mathrm{~mm}$ between the two sides and the outermost medial and lateral bone contour was applied. Subsequently, the centroids of the resulting oblong and of the superimposed bone were matched and the five VOIs were automatically placed along the medial-lateral axis intersecting the two centroids. The VOI 3, the center-reference, was placed on top of the centroid. The distance between the sides of the quadratic VOIs 1 and 5 and the corresponding edges of the rectangle were kept in the order of $1.2 \mathrm{~mm}$. VOIs 2 and 4 were placed at half the distance between VOI 1 and 5 and VOI 3, respectively. After positioning in the coronal plane, the extent along the coronal direction of these VOIs was defined. Using the previously derived sagittal plane, the VOIs were manually placed adjacent to the joint surface [see Fig. 1b]. Subsequently, the resulting coordinates were translated to the originally tomographic data and automatically extended over the successive 


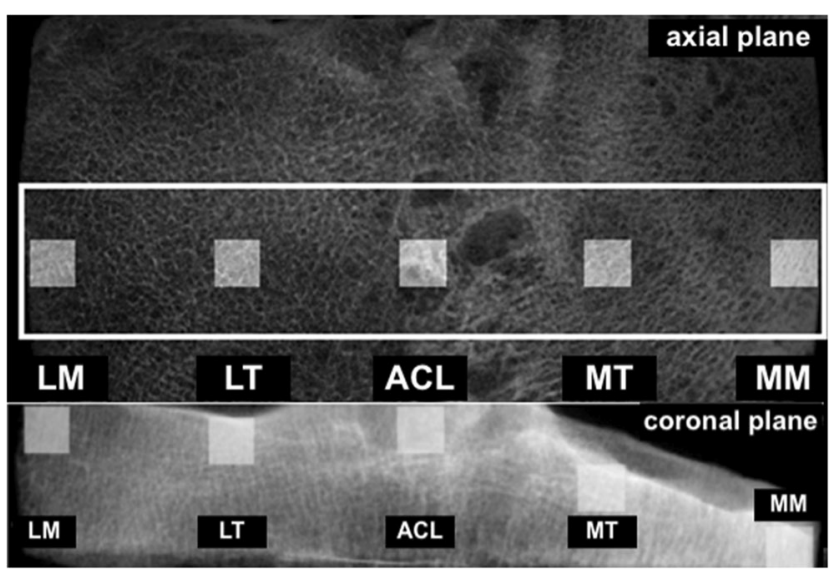

Fig. 1 a Axial plane of a representative bone sample. A white rectangle was marked (white box) in the coronal plane wherein the locations of the five VOIs were automatically derived. b Coronal plane of a representative bone sample. As a second step, the first coronal slice of each predefined VOI was manually adjusted in the sagittal plane and automatically extended over the range of $4 \mathrm{~mm}$

coronal slices. However, in case the VOI jutted out over the bone sample, the proximal end of the corresponding VOI was placed two slices above the margin of that sample. Finally, these semi-automatically derived volumes of each sample were transferred to commercially available software for microstructural analysis (see below).

\section{Analysis of bone microstructural parameters}

Siemens Inveon Research Workplace 3.0 (Siemens Medical Solutions Inc., Malvern, PA, USA) was used to analyze the following bone micro-structural parameters: Bone fraction volume (bone volume/total volume (BV/TV)), bone surface area/bone volume (BSA/BV), trabecular thickness (Tr.Th.), trabecular number (Tr.N.), trabecular spacing (Tr.Sp.), and trabecular pattern factor (Tb. Pf.).

\section{Statistics}

Data are presented as means and standard deviation (SD). Differences between locations (MM, medial meniscus; MT, medial tibia plateau; LM, lateral meniscus; LT lateral tibia plateau; ACL anterior cruciate ligament) were analyzed using ANOVA for repeated measurements. A $p$ value of $<0.05$ was considered to be statistically significant. Due to the exploratory character of the study, no $p$ value adjustment for multiple testing was applied. Statistical analysis was performed with IBM-SPSS Statistics 22 (SPSS Inc., Chicago, Illinois; 2013).

\section{Ethics}

The conduction of the study was approved by the Institutional Review Board of the Medical University of Graz (EK-Nr.: 25203 ex 12/13). The representative material and patient demographic data were collected after obtaining written informed consent. In all cases, acquisition of samples complied with the 1975 Declaration of Helsinki.

\section{Results}

Thirty proximal tibial specimens of 30 patients ( 20 women and 10 men) with late-stage knee OA were included in this study. The mean age of all patients in years was $70.4 \pm 9.9$. Women were older $(74.3 \pm 6.9$ years $)$ than men $(62.5 \pm 10.6$ years) $(p=0.003$ ). BMI in all patients was $29.5 \pm 3.7$ (women $30.1 \pm 3.7$, men $28.3 \pm 3.7 ; p=0.32$ ). There were 5 patients with osteoporosis $(16.6 \%$, women $n=5)$ and 8 patients with osteopenia $(26.7 \%$, women $n=6$, men $n=2)$. But both osteoporosis and osteopenia did not have any effect on the results, especially on BV/TV. For more patients' demographic data see Table 1.

\section{Knee Society Score}

The mean Knee Society Score (KSS) for all patients was 52.4 \pm 11.6 (similar in women $51.7 \pm 12.8$ compared to men $53.7 \pm$ 9.0, $p=0.39$ ) and the mean Knee Society Function Score was $44.0 \pm 12.2$ (also similar in women $43.8 \pm 14.3$ compared to men $44.5 \pm 6.9, p=0.17$ ), see Table 1 .

\section{Cartilage damage assessed by MRI}

The mean grade of cartilage damage in the medial compartment was $3.5 \pm 0.7$ (women $3.4 \pm 0.7$, men $3.7 \pm 0.6 ; p=$ $0.195)$ compared to the mean grade of cartilage damage of $2.28 \pm 0.8$ in the lateral compartment (women $2.3 \pm 0.9$, men $2.3 \pm 0.8 ; p=0.358$ ). The degree of cartilage damage analyzed in various locations can be seen in Table 1 .

\section{$\mu C T$ imaging}

The results of the $\mu \mathrm{CT}$ analysis can be seen in Table 2 . The mean $\mathrm{BV} / \mathrm{TV}$ in the medial compartment was significantly higher compared to the mean $\mathrm{BV} / \mathrm{TV}$ in the lateral compartment $(62.07 \pm 12.53$ vs. $52.57 \pm 13.33 ; p=0.007)$. The submeniscal mean BV/TV in the medial compartment was significantly higher compared to submeniscal mean BV/TV in the lateral compartment $(56.76 \pm 12.8$ vs. $47.36 \pm 14.97 ; p=$ $0.015)$. There was a significantly lower BV/TV in the medial submeniscal subchondral bone compared to the subchondral $\mathrm{BV} / \mathrm{TV}$ from the medial tibial plateau compartment $(p=$ 0.041). There was a significant difference in the lateral submeniscal subchondral BV/TV compared to the subchondral BV/TV from the lateral tibial plateau compartment $(p=0.024)$. The BV/TV in all weight-bearing locations (medial meniscus, medial tibial plateau, lateral meniscus, 
Table 1 Patients' demographic data

All patients $(n=30)$ (mean and SD) Female patients $(n=20)($ mean and SD) Male patients $(n=10)$ (mean and SD)

\begin{tabular}{llll}
\hline Age (in years) & $70.4 \pm 9.9$ & $74.3 \pm 6.9$ & $62.5 \pm 10.6$ \\
Height (in cm) & $166.7 \pm 7.7$ & $162.8 \pm 4.9$ & $174.6 \pm 6.3$ \\
Weight (in Kg) & $82.0 \pm 11.3$ & $79.8 \pm 10.4$ & $28.3 \pm 3.7$ \\
BMI & $29.5 \pm 3.7$ & $30.1 \pm 3.7$ & $53.7 \pm 9.0$ \\
Knee Society Score & & & $44.5 \pm 6.9$ \\
Knee Score & $52.4 \pm 11.6$ & $51.7 \pm 12.8$ & $3.7 \pm 0.6$ \\
Knee Society Function Score & $44.0 \pm 12.2$ & $43.8 \pm 14.3$ & $3.3 \pm 0.6$ \\
Degree of cartilage damage & & & $2.3 \pm 0.8$ \\
CD med. & $3.5 \pm 0.7$ & $3.4 \pm 0.7$ & $2.0 \pm 0.5$ \\
CD sm med. & $3.3 \pm 0.7$ & $3.3 \pm 0.8$ & $2.3 \pm 0.9$ \\
CD lat. & $2.3 \pm 0.8$ & $1.7 \pm 0.8$ & \\
CD sm lat. & $1.8 \pm 0.6$ & & \\
\hline
\end{tabular}

Legend: $C D$ cartilage damage according to Yulish et al., sm submeniscal, med medial, lat lateral

lateral tibial plateau) was significantly higher compared to the non-weight-bearing reference point below the ACL $(p<$ 0.001) [Table 2].

Among the microarchitectural parameters, there were some statistically significant differences $(p=0.05)$ between men and women: In MT trabecular thickness (men 0.41 vs. women 0.44; $p=0.024$ ) and trabecular number (men 1.55 vs. women $1.60 ; p=$ 0.026 ) were higher in women compared to men, whereas the trabecular pattern factor (men 5.82 vs. women $5.16 ; p=0.01$ ) was higher in men than in women. In LT BV/TV (men 0.45 vs. women $0.55 ; p=0.044$ ) was higher in women, but trabecular pattern factor (men -2.76 vs. women $-4.84 ; 0.006$ ) was higher in men. Trabecular number in the medial (men 1.55 vs. women $1.60 ; p=0.038$ ) was higher in men than in women, and trabecular pattern factor in the lateral compartment (men -2.30 vs. women -4.37 ; $p=0.035$ ) was higher in men.

\section{Meniscus integrity}

Thirteen of the medial menisci were intact, whereas 17 were either sub-luxated or luxated. On the other hand, 24 of the lateral menisci were intact, whereas six were either subluxated or luxated. In intact menisci, there was a significantly lower subchondral BV/TV compared to subluxated or luxated menisci in the medial $(p=0.020)$ and lateral compartment $(p=$ $0.005)$ [Figs. 2 and 3].

\section{Knee joint alignment}

There was a varus alignment in 22 patients with a mean of $6.3^{\circ}$ $\pm 4.5^{\circ}$ deviation. Six patients had a valgus alignment with a mean of $7.0^{\circ} \pm 4.4^{\circ}$ deviation. Two patients had an orthograde axis. Varus alignment resulted in a significantly higher subchondral $\mathrm{BV} / \mathrm{TV}$ in the medial compartment than valgus, whereas valgus alignment had a significantly higher subchondral $\mathrm{BV} / \mathrm{TV}$ in the lateral compartment $(p=0.011)$ [Fig. 4].

\section{Cartilage damage}

There was a significant correlation between the degree of cartilage damage and $\mathrm{BV} / \mathrm{TV}(p<0.001), \mathrm{BSA} / \mathrm{BV}(p=0.008)$, trabecular thickness $(p=0.008)$, trabecular separation $(p=$ $0.001)$, and trabecular pattern factor $(p=0.005)$ in MT. The distribution of $\mathrm{BV} / \mathrm{TV}$ of all measured locations in respect to cartilage damage in male and female patients is shown in Fig. 5.

\section{Discussion}

Recently, subchondral bone shifted into the focus as a possible cause of OA initiation and progression $[3,7]$ and subsequently as a potential treatment target [3]. Increased bone remodelling in the subchondral bone can be found in early-stage disease [16-19], leading to bone thickening as OA progresses, whereas in late-stage disease, the rate of remodelling decreases. In tissue specimens of late-stage hip OA with focal cartilage damage, increased subchondral bone density was seen in histomorphometry as well as in microstructural analyses by $\mu \mathrm{CT}[20]$.

To our knowledge, this is the first study on subchondral bone changes conducted in patients with advanced stage of osteoarthritis of the knee. New is also the combination of the approach and the methods used: (1) Before the total knee arthroplasty, we had x-rays and MRI done to investigate malalignment, cartilage damage, and meniscus integrity. (2) Due to the resection technique in total knee arthroplasty 


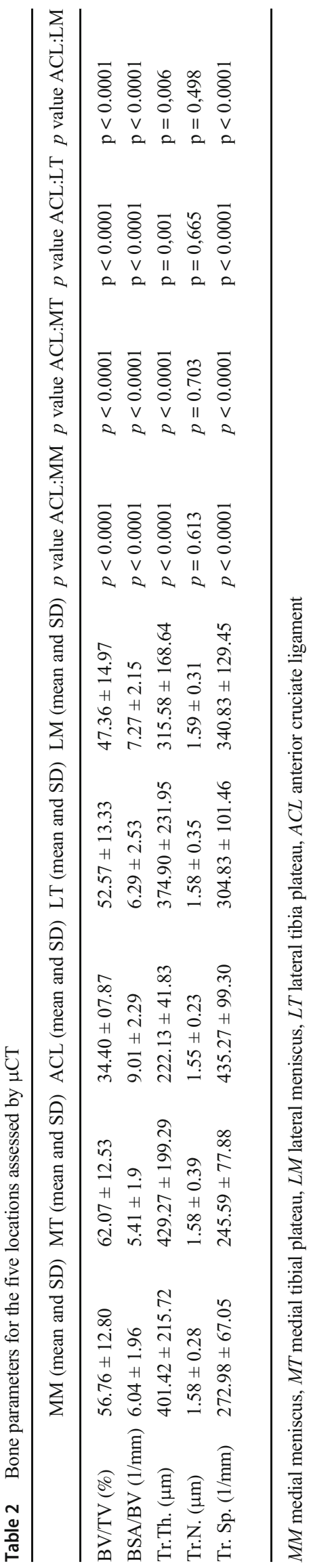

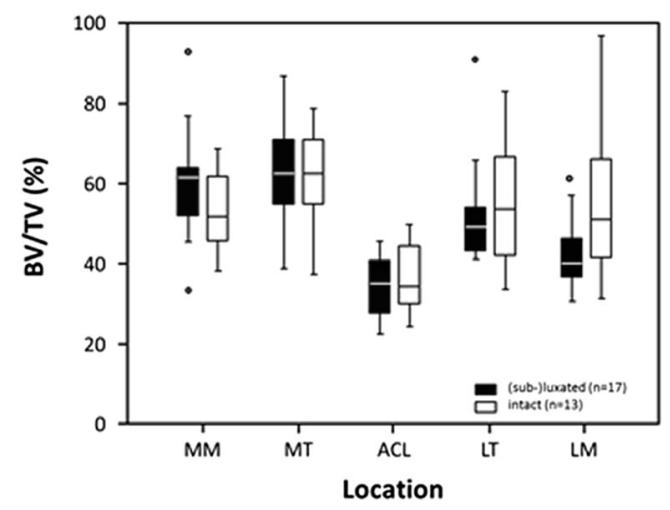

Fig. 2 Boxplots of subchondral BV/TV in the 5 analyzed locations (MM, medial meniscus; MT, medial tibial compartment; ACL, anterior cruciate ligament; LT, lateral tibial compartment; LM, lateral meniscus) in respect to intact (white boxes) and subluxated or luxated (black boxes) medial menisci.. Circles are outliners

(TKA) where the tibial plateau is resected in one piece, we were able to examine different subchondral bone areas within the tibial plateau. (3) We analyzed the subchondral bone in total tibial plateaus by using microCT. Segmentation of the initial scan was done using semi-automatic segmentation software. (4) The use of the above mentioned approach and methods made possible a comparison of subchondral bone changes and articular cartilage damage, meniscus integrity, and knee joint alignment.

In a biomechanical setting, results showed that cartilage of OA knees had a lower force attenuation compared to physiological cartilage [9]. We can expect that with severe cartilage damage subchondral BV/TV will be increased. The results of this study showed that the degree of cartilage damage varies within the knee compartments and that these changes are associated with subchondral bone changes. In areas of higher cartilage damage, a significantly increased subchondral BV/ $\mathrm{TV}$ could be identified. The highest BV/TV was seen in the medial subchondral tibial compartment, where also the highest degree of cartilage damage could be assessed. These

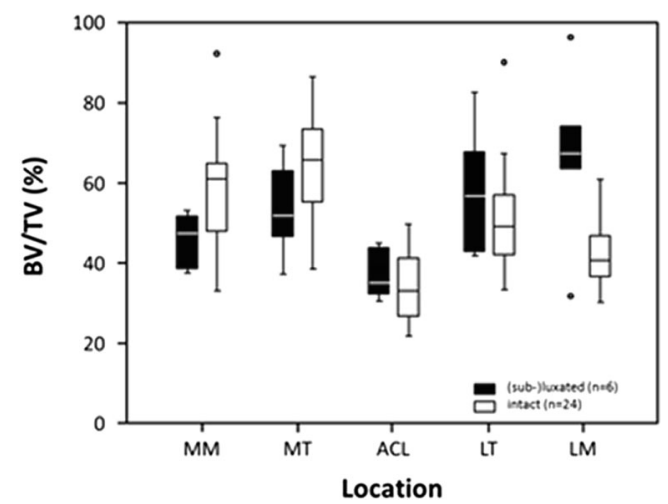

Fig. 3 Boxplots of subchondral BV/TV in the 5 analyzed locations (MM, medial meniscus; MT, medial tibial compartment; ACL, anterior cruciate ligament; LT, lateral tibial compartment; LM, lateral meniscus) in respect to intact (white boxes) and subluxated or luxated (black boxes ) lateral menisci 


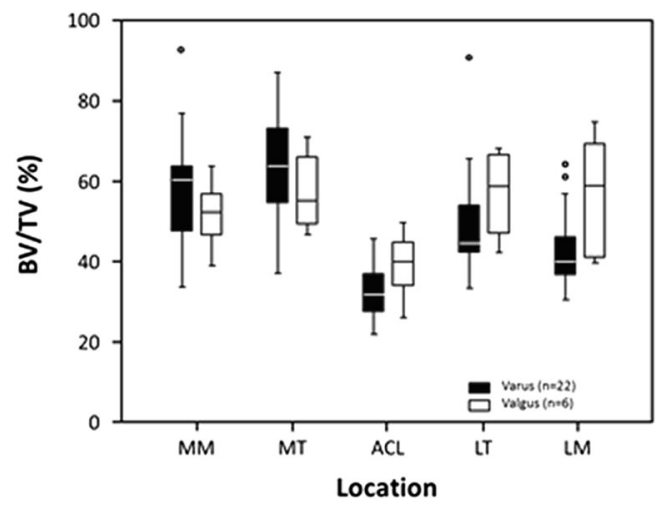

Fig. 4 Boxplots of subchondral BV/TV in 5 analyzed locations (MM, medial meniscus; MT, medial tibial compartment; ACL, anterior cruciate ligament; LT, lateral tibial compartment; LM, lateral meniscus) in respect to knee joint alignment. Varus (black boxes) and valgus alignment (white boxes).

results provide evidence that subchondral bone changes in $\mathrm{OA}$ are secondary processes. These findings are supported by the results of a study on femoral heads in patients with hip OA [8].

Subchondral bone changes were more pronounced in patients' knees that showed structural changes of the menisci or malalignment than in knees of patients who did not have any structural or mechanical alterations. We therefore conclude that the cartilage damages are due to the loss of the meniscus' integrity or malalignment and therefore can be considered as "secondary."

We also looked at meniscal integrity by using MRI. Intact meniscal structures reduce the mechanical impacts at the proximal tibia and have therefore been considered to be beneficial for cartilage integrity and to prevent OA progression. In this study, the sub-meniscal subchondral bone had significantly lower BV/ TV compared to weight-bearing locations centrally at the medial and lateral tibial compartment. This supports the non-surgical management of degenerative, but structurally intact, menisci. On the other hand, the results also support the indication of surgical intervention in case of luxation or subluxation of menisci $[12,13]$.

Another risk factor for the initiation and progression of OA is knee joint malalignment [21]. The majority of patients studied had a varus alignment and a higher subchondral BV/TV in the medial compartment compared to the lateral one. This might support the role of corrective osteotomy to prevent OA progression in both cartilage and subchondral bone.

Due to the study design, as patients scheduled for surgery were recruited chronologically, more women than men were included in this study, and women were also older (mean age $74.3+-6.9$ versus $62.5+-10.6)$. Concerning differences between men and women, microarchitectural parameters proved statistically significantly different $(p<0.05)$ in MT and LT, the medial and lateral compartment. BV/TV in LT, trabecular thickness in MT, and trabecular number in both MT and in the medial compartment was higher in women compared to men, whereas the trabecular pattern factor in MT, LT, and the lateral compartment was higher in men compared to women.

Microstructural parameters, especially BV/TV, were not affected by osteoporosis or osteopenia. Although there were five patients with osteoporosis and seven with osteopenia, it seems that mechanical factors like cartilage damage, meniscus' structural integrity, and knee joint alignment in local areas plays a more important role than a systemic disease.

The strength of this study is that human ex vivo knee OA specimens were studied. The majority of previous studies investigating the role of subchondral bone in OA were conducted in an experimental setting using animals $[3,19]$. In humans, studies were performed mainly at the hip. In most experimental studies published previously, knee OA initiation and
Fig. 5 Distribution of BV/TV in respect to cartilage damage in male and female patients

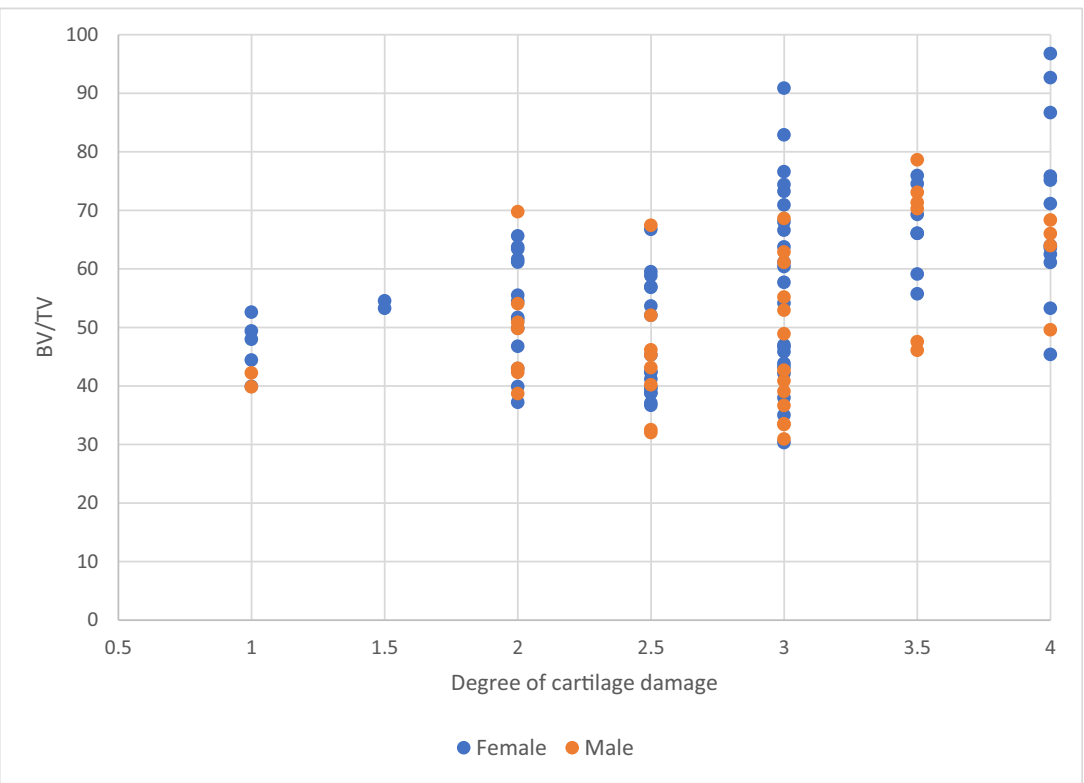


progression were induced by either sectioning the ACL or PCL or collateral ligaments or complete meniscectomy to produce OA $[3,19]$. So, these studies induced a secondary OA of the knee. In this study, patients had a continuous natural progression of disease resulting in a primary OA of the knee.

Another strength of this study is the use of a semiautomatic segmentation software for identifying the VOI in the proximal tibial scans. This avoids subjective influence on selection of the studied region. Therefore, results are comparable on a more objective level. An auto-segmented VOI size of $4 \mathrm{~mm}^{3}$ was chosen, compared to manually extracted core cylinders that analyzed specimens of up to $10 \mathrm{~mm}$ longitudinal size. The most dramatic and homogeneous changes of subchondral bone density in OA are described within the first $6 \mathrm{~mm}$ below the cartilage [22]. So, more realistic values could be reached compared to other studies that used cores to extract cylinders of $10 \mathrm{~mm}$ length manually. Furthermore, by using auto-segmentation in a whole scan of a specimen, the risk of artifacts is also reduced as parts close to the cutting edge are not being destroyed compared to manual extraction [23]. The same segmentation process to identify the various VOIs in each tibial plateau was applied in all specimens. Due to the anatomical differences such as malalignment, the resection level is close to the joint line. In such cases, the volume of a VOI was lower compared to a resection that was $10 \mathrm{~mm}$ below the joint line.

A weakness of the study might be the sample size of 30 specimens. However, this sample number is comparable or even higher than other studies using $\mu \mathrm{CT}[3,8]$. Another limiting factor might be the evaluation of cartilage degeneration by MRI using a semiquantitative method as described by Yulish et al. [8]. On the other hand, it has been shown that evaluation of cartilage damage by MRI correlates well with histopathologic analyses [24].

\section{Conclusions}

Concluding, we could show significant differences of subchondral bone microstructural parameters in respect to cartilage damage, meniscus' structural integrity, and knee joint alignment. Therefore, subchondral microstructural bone changes seem to be a secondary process in late-stage OA of the knee caused by mechanical changes, and the proper management of these changes might prevent subchondral bone changes and therefore prevent or slow the progression of OA.

Acknowledgements The study was supported by a Bank Austria Visiting Scientist grant of the Medical University of Graz.

Funding information Open access funding provided by Medical University of Graz.

\section{Compliance with ethical standards}

Competing interest None.
Open Access This article is licensed under a Creative Commons Attribution-NonCommercial 4.0 International License, which permits any non-commercial use, sharing, adaptation, distribution and reproduction in any medium or format, as long as you give appropriate credit to the original author(s) and the source, provide a link to the Creative Commons licence, and indicate if changes were made. The images or other third party material in this article are included in the article's Creative Commons licence, unless indicated otherwise in a credit line to the material. If material is not included in the article's Creative Commons licence and your intended use is not permitted by statutory regulation or exceeds the permitted use, you will need to obtain permission directly from the copyright holder. To view a copy of this licence, visit http:// creativecommons.org/licenses/by-nc/4.0/.

\section{References}

1. Murray CJL, Lopez AD (1996) The global burden of disease. World Health Organisation, Geneva

2. Goldring SR, Goldring MB (2010) Bone and cartilage in osteoarthritis: is what's best for one good or bad for the other? Arthritis Res Ther 12:143

3. Burr DB, Gallant MA (2012) Bone remodelling in osteoarthritis. Nat Rev Rheumatol 8:665-673

4. Burr DB, Radin EL (2003) Microfractures and microcracks in subchondral bone: are they relevant to osteoarthrosis? Rheum Dis Clin North Am 29:675-685

5. Fazzalari N, Parkinson IH (1997) Fractal properties of subchondral cancellous bone in severe osteoarthritis of the hip. J Bone Miner Res 12:632-640

6. Lories RJ, Luyten FP (2011) The bone-cartilage unit in osteoarthritis. Nat Rev Rheumatol 7:43e9

7. Hannan MT, Anderson JJ, Zhang Y, Levy D, Felson DT (1993) Bone mineral density and knee osteoarthritis in elderly men and women. The Framingham Study. Arthritis Rheum 36:1671-1680

8. Zhang ZM, Li ZC, Jiang LS, Jiang SD, Dai LY (2010) Micro-CT and mechanical evaluation of subchondral trabecular bone structure between postmenopausal women with osteoarthritis and osteoporosis. Osteoporos Int 21:1383-1390

9. Hoshino A, Wallace WA (1987) Impact-absorbing properties of human knee. J Bone Joint Surg Br 69-B:807-811

10. Roemhildt ML, Beynnon BD, Gardner-Morse M, Badger G, Grant C (2012) Changes induced by chronic in vivo load alteration in the tibiofemoral joint of mature rabbits. J Orthop Res 30:1413-1422

11. Shiomi T, Nishii T, Tamura S, Tanaka H, Murase K, Yoshikawa H, Sugano N (2012) Influence of medial meniscectomy on stress distribution of the femoral cartilage in porcine knees: a $3 \mathrm{D}$ reconstructed T2 mapping study. Osteoarthritis Cartilage 20:1383-1390

12. Holzer LA, Leithner A, Holzer G (2013) Surgery versus physical therapy for meniscal tear and osteoarthritis. N Engl J Med 369:677

13. Katz JN, Brophy RH, Chaisson CE, de Chaves L, Cole BJ, Dahm DL, Donnell-Fink LA, Guermazi A, Haas AK, Jones MH, Levy BA, Mandl LA, Martin SD, Marx RG, Miniaci A, Matava MJ, Palmisano J, Reinke EK, Richardson BE, Rome BN, SafranNorton CE, Skoniecki DJ, Solomon DH, Smith MV, Spindler KP, Stuart MJ, Wright J, Wright RW, Losina E (2013) Surgery versus physical therapy for a meniscal tear and osteoarthritis. $\mathrm{N}$ Engl J Med 368:1675-1684

14. Insall JN, Dorr LD, Scott RD, Scott WN (1989) Rationale of the Knee Society clinical rating system. Clin Orthop Relat Res 248:1314 
15. Yulish BS, Montanez J, Goodfellow DB, Bryan PJ, Mulopulos GP, Modic MT (1987) Chondromalacia patellae: assessment with MR imaging. Radiology 164:763-766

16. Intema F, Sniekers YH, Weinans H, Vianen ME, Yocum SA, Zuurmond AM, DeGroot J, Lafeber FP, Mastbergen SC (2010) Similarities and discrepancies in subchondral bone structure in two differently induced canine models of osteoarthritis. J Bone Miner Res 25:1650-1657

17. Mansell JP, Tarlton JF, Bailey AJ (1997) Biochemical evidence for altered subchondral bone collagen metabolism in osteoarthritis of the hip. Br J Rheumatol 36:16-19

18. Reichenbach S, Guermazi A, Niu J, Neogi T, Hunter DJ, Roemer FW, McLennan CE, Hernandez-Molina G, Felson DT (2008) Prevalence of bone attrition on knee radiographs and MRI in a community-based cohort. Osteoarthritis Cartilage 16:1005-1010

19. Sniekers YH, Intema F, Lafeber FP, van Osch GJ, van Leeuwen JP, Weinans H, Mastbergen SC (2008) A role for subchondral bone changes in the process of osteoarthritis; a micro-CT study of two canine models. BMC Musculoskelet Disord 9:20

20. Kumarasinghe DD, Perilli E, Tsangari H, Truong L, Kuliwaba JS, Hopwood B, Atkins GJ, Fazzalari NL (2010) Critical molecular regulators, histomorphometric indices and their correlations in the trabecular bone in primary hip osteoarthritis. Osteoarthritis Cartilage 18:1337-1344

21. Tanamas S, Hanna FS, Cicuttini FM, Wluka AE, Berry P, Urquhart DM (2009) Does knee malalignment increase the risk of development and progression of knee osteoarthritis? A systematic review. Arthritis Rheum 15:559-567

22. Patel V, Issever AS, Burghardt A, Laib A, Ries M, Majumdar S (2003) MicroCT evaluation of normal and osteoarthritic bone structure in human knee specimens. J Orthop Res 21:6-13

23. Fajardo RJ, Cory E, Patel ND, Nazarian A, Laib A, Manoharan RK, Schmitz JE, DeSilva JM, MacLatchy LM, Snyder BD, Bouxsein ML (2009) Specimen size and porosity can introduce error into microCT-based tissue mineral density measurements. Bone 44: 176-184

24. Trattnig S, Huber M, Breitenseher MJ, Trnka HJ, Rand T, Kaider A, Helbich T, Imhof H, Resnick D (1998) Imaging articular cartilage defects with 3D fat-suppressed echo planar imaging: comparison with conventional 3D fat-suppressed gradient echo sequence and correlation with histology. J Comput Assist Tomogr 22:8-14

Publisher's note Springer Nature remains neutral with regard to jurisdictional claims in published maps and institutional affiliations. 